\title{
PARABOLIC CURVES FOR DIFFEOMORPHISMS IN $\left(\mathbb{C}^{2}, 0\right)$
}

\author{
F. E. BROCHERO MARTÍNEZ, F. CANO, AND L. LÓPEZ-HERNANZ
}

\begin{abstract}
We give a simple proof of the existence of parabolic curves for tangent to the identity diffeomorphisms in $\left(\mathbb{C}^{2}, 0\right)$ with isolated fixed point.
\end{abstract}

\section{INTRODUCTION}

Let $F$ be a tangent to the identity diffeomorphism of $\left(\mathbb{C}^{n}, 0\right)$. A parabolic curve for $F$ is an injective holomorphic map $\varphi: \Omega \rightarrow \mathbb{C}^{n}$, where $\Omega$ is a simply connected domain in $\mathbb{C}$ with $0 \in \partial \Omega$ such that

(1) $\varphi$ is continuous at the origin, and $\varphi(0)=0$.

(2) $F(\varphi(\Omega)) \subset \varphi(\Omega)$ and $F^{\circ k}(p)$ converges to 0 when $k \rightarrow+\infty$, for $p \in \varphi(\Omega)$. We say that $\varphi$ is tangent to $[v] \in \mathbb{P}^{n-1}$ if $[\varphi(\zeta)] \rightarrow[v]$ when $\zeta \rightarrow 0$. Let us write $F(z)=z+P_{k}(z)+P_{k+1}(z)+\cdots$, where $P_{j}$ is a $n$-dimensional vector of homogeneous polynomials of degree $j$, and $P_{k}(z) \not \equiv 0$. A characteristic direction for $F$ is a point $[v] \in \mathbb{P}^{n-1}$ such that $P_{k}(v)=\lambda v$, for some $\lambda \in \mathbb{C}$; it is nondegenerate if $\lambda \neq 0$. The integer $\operatorname{ord}(F):=k \geq 2$ is the tangency order of $F$ at 0 .

The following theorem is analogous to Briot and Bouquet's theorem [3] for diffeomorphisms of $\left(\mathbb{C}^{n}, 0\right)$.

Theorem 1.1 (Hakim [6]). Let $F$ be a tangent to the identity germ of diffeomorphism of $\left(\mathbb{C}^{n}, 0\right)$. For any nondegenerate characteristic direction $[v]$ there exist $\operatorname{ord}(F)-1$ disjoint parabolic curves tangent to $[v]$ at the origin.

When $n=2$, Abate proved that the nondegeneracy condition can be dismissed.

Theorem 1.2 (Abate 1, 2]). Let $F$ be a tangent to the identity germ of diffeomorphism of $\left(\mathbb{C}^{2}, 0\right)$ such that 0 is an isolated fixed point. Then there exist $\operatorname{ord}(F)-1$ disjoint parabolic curves for $F$ at the origin.

This theorem is analogous to Camacho-Sad's theorem [4] of existence of invariant curves for holomorphic vector fields. We show in this note that the analogy is deeper enough to prove theorem 1.2 in a simple way starting with Hakim's theorem.

\section{Exponential Operator and Blow-up transformation}

Let $\hat{\mathfrak{X}}_{2}\left(\mathbb{C}^{2}, 0\right)$ be the module of formal vector fields $X=a(x, y) \frac{\partial}{\partial x}+b(x, y) \frac{\partial}{\partial y}$ of order $\geq 2$, i.e., $\min \{\nu(a), \nu(b)\} \geq 2$. We denote by $\widehat{\operatorname{Diff}}_{1}\left(\mathbb{C}^{2}, 0\right)$ the group of tangent to the identity formal diffeomorphisms $F(x, y)=(x+p(x, y), y+q(x, y))$ where

Date: September 22, 2018.

2000 Mathematics Subject Classification. 32H02, 32H50, 37F99.

The first author was supported by CAPES, Brazil, Process: BEX3083/05-5.

The third author was supported by FPU, Spain, Process:AP2005-3784. 
$\min \left\{\nu(p(x, y)), \nu(q(x, y)\} \geq 2\right.$. Let us denote by $\mathfrak{X}_{2}\left(\mathbb{C}^{2}, 0\right)$ and by $\operatorname{Diff}_{1}\left(\mathbb{C}^{2}, 0\right)$ the convergent elements of $\hat{\mathfrak{X}}_{2}\left(\mathbb{C}^{2}, 0\right)$ and $\widehat{\operatorname{Diff}}_{1}\left(\mathbb{C}^{2}, 0\right)$ respectively.

Let $X \in \widehat{\mathfrak{X}}_{2}\left(\mathbb{C}^{2}, 0\right)$. The exponential operator of $X$ is the application $\exp t X$ : $\mathbb{C}[[x, y]] \rightarrow \mathbb{C}[[x, y, t]]$ defined by the formula

$$
\exp t X(g)=\sum_{j=0}^{\infty} \frac{t^{j}}{j !} X^{j}(g)
$$

where $X^{0}(g)=g$ and $X^{j+1}(g)=X\left(X^{j}(g)\right)$. Note that, since $\nu\left(X^{j}(g)\right) \geq j+\nu(g)$, we can substitute $t=1$ to get the element $\exp X(g) \in \mathbb{C}[[x, y]]$. Moreover, $\exp t X$ gives a homomorphism of $\mathbb{C}$-algebras, in particular, we have

$$
\exp t X(f g)=\exp t X(f) \exp t X(g) .
$$

We get also

Proposition 2.1. The application

$$
\begin{aligned}
\operatorname{Exp}: \widehat{\mathfrak{X}}_{2}\left(\mathbb{C}^{2}, 0\right) & \rightarrow \widehat{\operatorname{Diff}}_{1}\left(\mathbb{C}^{2}, 0\right) \\
X & \mapsto(\exp X(x), \exp X(y))
\end{aligned}
$$

is a bijection.

Proof: Let $G(x, y)=\left(x+\sum_{n=2}^{\infty} p_{n}(x, y), y+\sum_{n=2}^{\infty} q_{n}(x, y)\right)$ and $X=\sum_{n=2}^{\infty}\left(a_{n}(x, y) \frac{\partial}{\partial x}+\right.$ $\left.b_{n}(x, y) \frac{\partial}{\partial y}\right)$. The identity $\operatorname{Exp}(X)=G$ is equivalent to

$$
\begin{aligned}
& p_{m+1}=a_{m+1}+H T_{m+1}\left(\sum_{j=2}^{m} \frac{1}{j !} X_{m}^{j}(x)\right) \\
& q_{m+1}=b_{m+1}+H T_{m+1}\left(\sum_{j=2}^{m} \frac{1}{j !} X_{m}^{j}(y)\right),
\end{aligned}
$$

where $X_{m}=\sum_{n=2}^{m}\left(a_{n}(x, y) \frac{\partial}{\partial x}+b_{n}(x, y) \frac{\partial}{\partial y}\right)$, and $H T_{m+1}(h)$ is the homogeneous term of $h$ of order $m+1$. These equations determine univocally $X$ if $G$ is given. $\square$

In general, $X$ may be not convergent for certain convergent $G$. The formal vector field $X$ such that $G=\operatorname{Exp}(X)$ is called the infinitesimal generator of $G$. Note that $\operatorname{ord}(G)=\nu(X)$. If $k=\nu(X)$, then $a_{k}=p_{k}$ and $b_{k}=q_{k}$, thus the characteristic directions of $F$ correspond to the points of the tangent cone of $X$. Moreover, if $X=f X^{\prime}$ with $X^{\prime} \in \widehat{\mathfrak{X}}\left(\mathbb{C}^{2}, 0\right)$ and $f \in \mathbb{C}[[x, y]]$ then $\operatorname{Exp}(X)(x, y)=$ $(x+f(x, y) p(x, y), y+f(x, y) q(x, y))$. The converse statement follows by a process similar to the proof of proposition 2.1 In particular, 0 is an isolated singular point of $X$ if and only if 0 is an isolated fixed point of $F$. In the case $f(x, y)=x^{k}$, and $S=(x=0)$ invariant by $X^{\prime}$, Camacho-Sad's index of $X$ at 0 along $S$ is exactly Abate's residual index of $F$ at 0 along $S$.

Now, let $\pi:(M, D) \rightarrow\left(\mathbb{C}^{2}, 0\right)$ be the blow up of $\mathbb{C}^{2}$ at the origin, where $D=$ $\pi^{-1}(0)=\mathbb{P}^{1}$, thus each characteristic direction determines a point of $D$.

Proposition 2.2. Let $F \in \operatorname{Diff}_{1}\left(\mathbb{C}^{2}, 0\right)$. There exists a unique germ of diffeomorphism $\tilde{F}$ in $(M, D)$ such that $\pi \circ \tilde{F}=F \circ \pi$ and $\left.\tilde{F}\right|_{D}=\left.i d\right|_{D}$. Moreover, the germ $\tilde{F}_{p}$ has order $\geq \operatorname{ord}(F)$ for any characteristic direction $p \in D$ and hence $\tilde{F}_{p} \in \operatorname{Diff}_{1}(M, p)$. 
Proof: Let $F(x, y)=\left(x+p_{k}(x, y)+\cdots, y+q_{k}(x, y)+\cdots\right)$ where $k=\operatorname{ord}(F) \geq 2$. We have two charts of $M=U_{1} \cup U_{2}$ such that $\left.\pi\right|_{U_{1}}: U_{1} \rightarrow \mathbb{C}^{2}$, is defined by $\pi(x, v)=(x, x v)$ and $\left.\pi\right|_{U_{2}}: U_{2} \rightarrow \mathbb{C}^{2}$, is defined by $\pi(u, y)=(u y, y)$. We define $\tilde{F}$ in the first chart as

$$
\begin{aligned}
\tilde{F}(x, v) & =\pi^{-1} \circ F \circ \pi(x, v)=\left(x+p_{k}(x, x v)+\cdots, \frac{v x+q_{k}(x, x v)+\cdots}{x+p_{k}(x, x v)+\cdots}\right) \\
& =\left(x+x^{k}\left(p_{k}(1, v)+x(\cdots)\right), v+x^{k-1}\left(q_{k}(1, v)-v p_{k}(1, v)+x(\cdots)\right)\right)
\end{aligned}
$$

Observe that $\tilde{F}(0, v)=(0, v)$, thus any point of the divisor is fixed. Moreover, if $q_{k}\left(1, v_{0}\right)-v_{0} p_{k}\left(1, v_{0}\right)=0$ we have $d F\left(0, v_{0}\right)=I$, and thus for any characteristic direction $p=\left(0, v_{0}\right) \in D, \operatorname{ord}\left(\tilde{F}_{p}\right) \geq \operatorname{ord}(F)$.

Proposition 2.3. Let $X \in \widehat{\mathfrak{X}}_{2}\left(\mathbb{C}^{2}, 0\right)$. Let $\tilde{X}$ be the formal vector field in $(M, D)$ such that $D \pi \cdot \tilde{X}=X \circ \pi$. If $p$ is a point of the tangent cone of $X$ then $\tilde{X}_{p} \in$ $\widehat{\mathfrak{X}}_{2}(M, p)$.

Proof: Let $X=a(x, y) \frac{\partial}{\partial x}+b(x, y) \frac{\partial}{\partial y}$ with $a(x, y)=a_{k}(x, y)+\cdots, b(x, y)=$ $b_{k}(x, y)+\cdots$ and $k \geq 2$. Let $U_{1}$ and $U_{2}$ be two charts of $M=U_{1} \cup U_{2}$ as in the proposition above. Then $\tilde{X}$ is given in the chart $U_{1}$ by

$$
\begin{aligned}
\tilde{X}(x, v) & =a(x, x v) \frac{\partial}{\partial x}+\frac{b(x, x v)-v a(x, x v)}{\partial v} \\
& =x^{k}\left(a_{k}(1, v)+x(\cdots)\right) \frac{\partial}{\partial x}+x^{k-1}\left(\left(b_{k}(1, v)-v a_{k}(1, v)\right)+x(\cdots)\right) \frac{\partial}{\partial y}
\end{aligned}
$$

Now, if $p=\left(0, v_{0}\right) \in D$ is such that $b_{k}\left(1, v_{0}\right)-v_{0} a_{k}\left(1, v_{0}\right)=0$, then $\nu_{p}(a(x, x v)) \geq k$ and $\nu_{p}\left(\frac{b(x, x v)-v a(x, x v)}{x}\right) \geq k$ so $\tilde{X}_{p} \in \widehat{\mathfrak{X}}_{2}(M, p)$.

We say that $X$ is strictly non singular if $X=f X^{\prime}$, where $X^{\prime}$ is a non singular formal vector field. Otherwise, we say that $X$ is strictly singular. Note that in the above statement any strictly singular point of $\tilde{X}$ is in the tangent cone of $X$. Let us also recall that Seidenberg's reduction of singularities [7] is done by blowing-up at strictly singular points.

Lemma 2.4. Let $F \in \operatorname{Diff}_{1}\left(\mathbb{C}^{2}, 0\right)$ and $X \in \widehat{\mathfrak{X}}_{2}\left(\mathbb{C}^{2}, 0\right)$ such that $F=\operatorname{Exp}(X)$. Let $\tilde{X}$ be as in the proposition above. Then for any characteristic direction $p \in D$

$$
\tilde{F}_{p}=\operatorname{Exp}\left(\tilde{X}_{p}\right) .
$$

Proof: Let $U \simeq \mathbb{C}^{2}$ be a chart of $M$ such that $\left.\pi\right|_{U}: U \rightarrow \mathbb{C}^{2}$ is defined by $\pi(x, v)=(x, x v)$ and $p \in U \cap D=\{(0, v) \in U\}$ be a point on the divisor. Without lost of generality, applying a linear change of coordinates, we can suppose that $p=(0,0) \in U$. Since

$$
F(x, y)=\operatorname{Exp}(X)=(\exp X(x), \exp X(y)),
$$

using the definition of $\tilde{F}$, we have

$$
\begin{aligned}
\tilde{F}(x, v) & =\left(\exp X(x), \frac{\exp X(x v)}{\exp X(x)}\right)=\left(\exp X(x), \frac{\exp X(x) \exp X(v)}{\exp X(x)}\right) \\
& =(\exp X(x), \exp X(v))=(\exp \tilde{X}(x), \exp \tilde{X}(v))=\operatorname{Exp}\left(\tilde{X}_{p}\right)(x, v) .
\end{aligned}
$$

This ends the proof. 


\section{EXISTENCE OF PARABOLIC CURVES}

We need the following formal version of Camacho-Sad's theorem [4] whose proof goes exactly as the original one (see also [5]).

Theorem 3.1 (Camacho and Sad). Take $X \in \widehat{\mathfrak{X}}_{1}\left(\mathbb{C}^{2}, 0\right)$ with an isolated singularity at the origin. There is a desingularization morphism $\sigma:(\tilde{M}, \tilde{D}) \rightarrow\left(\mathbb{C}^{2}, 0\right)$ composition of a finite sequence of blow-ups with centers at strictly singular points and a point $p \in \tilde{D}$ satisfying the following property: There are local coordinates $(u, v)$ at $p$ such that $\tilde{D}_{p}=(u=0)$ and the transform $X^{*}$ of $X$ at $p$ is of the form:

$$
X^{*}(u, v)=u^{k}\left(\left(\lambda u+u^{2}(\cdots)\right) \frac{\partial}{\partial u}+(\mu v+u(\cdots)) \frac{\partial}{\partial v}\right)
$$

where $\lambda \neq 0$ and $\frac{\mu}{\lambda} \notin \mathbb{Q}_{>0}$.

Let us prove theorem 1.2 Take $X$ the infinitesimal generator of $F$, and consider $X^{*}$ and $p$ as in Camacho-Sad's Theorem. By lemma 2.4 we have

$$
F_{p}^{*}(u, v):=\operatorname{Exp}\left(X_{p}^{*}\right)=\left(u+\lambda u^{k+1}+O\left(u^{k+2}\right), v+\mu u^{k} v+O\left(u^{k+1}\right)\right)
$$

so $F_{p}^{*}$ is a diffeomorphism tangent to the identity, with $(1,0)$ as a nondegenerate characteristic direction. By Hakim's Theorem, there exist ord $(F)-1$ disjoint parabolic curves $\varphi_{j}: \Omega_{j} \rightarrow \tilde{M}$ for $F_{p}^{*}$ tangent to the direction $(1,0)$ at $p$. Since this direction is transversal to the divisor, it follows that $\overline{\varphi_{j}\left(\Omega_{j}\right)} \cap \tilde{D}=\{p\}$ and thereby $\pi \circ \varphi_{j}$ is also a parabolic curve for $F$.

Furthermore, according to J. Cano's proof [5] of Camacho-Sad's theorem, to find the points $p \in \tilde{D}$ that satisfy Camacho-Sad's theorem, it is enough to follow after the first blow up, the singularities with Camacho-Sad's index not in $\mathbb{Q} \geq 0$. Thus, there exist parabolic curves for any characteristic direction of $F$ that gives at the divisor Abate's residual index not in $\mathbb{Q}_{\geq 0}$ (see corollary 3.1. in [1]).

\section{REFERENCES}

[1] Abate, M., The residual index and the dynamics of holomorphic maps tangent to the identity, Duke Math. J. 107 (2001) 173-207

[2] Abate M., Bracci F., Tovena F., Index theorems for holomorphic self-maps. Ann. Math. 159 (2004), 819-864

[3] Briot, Bouquet, Recherches sur les propriétés les fonctions définies par des équations différentielles, J. Ecole Polytechnique, XXI, (1856) 133-198

[4] Camacho, C., Sad P., Invariant varieties through singularities of holomorphic vector fields Ann. of Math. 115 (1982) 579-595

[5] Cano, J., Construction of invariant curves for singular holomorphic vector fields, Proc. Amer. Math. Soc., 125 (1997) 2649-2650

[6] Hakim, M., Analytic transformations of $\left(\mathbb{C}^{p}, 0\right)$ tangent to the identity, Duke Math. J. 92 (1998) 403-428.

[7] Seidenberg, A., Reduction of singularities of the differential equation $A d Y=B d X$, Amer. J. Math. (1968) 248-269

Departamento de Matemática, UfmG, Belo Horizonte, MG, 30123-970, Brazil,

E-mail address: fbrocher@mat.ufmg.br

Departamento de Álgebra, Geometría y Topología, Universidad de Valladolid, Spain E-mail address: fcano@agt.uva.es

E-mail address: llopez@agt.uva.es 